\title{
CORRELATION OF HER2/NEU EXPRESSION AND HISTOPATHOLOGICAL GRADING AND STAGING OF COLORECTAL CARCINOMAS
}

\author{
Shameem Kasthuri Ummerali1, Suma Sarojini²
}

${ }^{1}$ Associate Professor, Department of Pathology, Government T D Medical College, Alappuzha, Kerala.

2Senior Resident, Department of Pathology, Government T D Medical College, Alappuzha, Kerala.

\begin{abstract}
\section{BACKGROUND}

Colorectal cancer ranks third among the most common leading causes of cancer-related mortality in the world. HER2/neu oncogene is one of four epidermal growth factor receptors. Overexpression of HER2/neu was studied in many cancer types such as breast and ovary. Its amplification is associated with poor prognosis. Most of the studies of HER2/neu expression in colorectal cancer showed wide ranges from 0 to $83 \%$.

Aim- This study was done to check the expression of HER2/neu in colon carcinoma and its correlation with the histological grades and stages.
\end{abstract}

\section{MATERIALS AND METHODS}

Study Subjects- 50 cases of histopathologically proven colon carcinoma satisfying inclusion and exclusion criteria. SettingDepartment of Pathology in Govt. T D Medical College, Alappuzha.

Period- One and half years (January 2014-June 2015).

Design- Descriptive study.

Statistical Analysis- Chi square test using software SPSS version 16.

\section{RESULTS}

The mean age in this study was 60.04 years. Male female ratio was 1:1. Sixty percent of the tumours were from colon and all the cases were adenocarcinoma NOS. Eighty percent of the cases were grade 1 tumours and stage B was the common stage noticed. Thirty two percent of the cases showed lymph node metastasis. Only $24 \%$ of cases showed HER $2 /$ neu positivity and maximum positive cases were from grade 1 tumours. Maximum number of HER2/neu positivity was given by stage B tumours and only $4 \%$ of cases with lymph node metastasis showed HER2/neu positivity. There was no statistically significant correlation found between HER2/neu expression with grade, stage and lymph node metastasis of the tumours.

\section{CONCLUSION}

There was no significant correlation between HER2/neu expression and tumour stages and grades.

\section{KEYWORDS}

Colorectal Cancer, HER2/neu, Immunohistochemistry.

HOW TO CITE THIS ARTICLE: Ummerali SK, Sarojini S. Correlation of HER2/NEU expression and histopathological grading and staging of colorectal carcinomas. J. Evolution Med. Dent. Sci. 2017;6(60):4407-4411, DOI: 10.14260/Jemds/2017/953

\section{BACKGROUND}

Colorectal cancer (CRC) is the third most common cancer and the leading cause of cancer death in men and women.[1] Chemotherapy has shown to be an efficient strategy for adjuvant therapy, but is still not capable of preventing recurrence in all patients. So there are numerous ongoing researches for alternative compounds to be used as adjuvant therapy.[2] A crucial step in this process is the identification of tumour specific proteins that can be targeted by these compounds. One of these targets is HER2/neu, which is primarily associated with breast cancer. HER2 is found to be upregulated in $20 \%-30 \%$ of all breast cancers. Trastuzumab, a monoclonal antibody against the extracellular

Financial or Other, Competing Interest: None.

Submission 30-05-2017, Peer Review 15-07-2017,

Acceptance 21-07-2017, Published 27-07-2017.

Corresponding Author:

Dr. Suma Sarojini,

Senior Resident, Department of Pathology,

Government T D Medical College,

Alappuzha-688005, Kerala.

E-mail: ssuma83@ymail.com

DOI: $10.14260 /$ jemds $/ 2017 / 953$

(c) $(7)$ domain of HER2, showed a dramatic improvement in survival as well as recurrence rate when used as adjuvant therapy after breast cancer surgery.[3,4]

After a $37 \%$ improvement in overall survival, this recently led to FDA-approval for the use of trastuzumab in HER2-positive metastatic gastric cancer.[5] Several studies evaluating HER2 in colorectal cancer resulted in a large debate because overexpression rates varied between zero and $84 \% .{ }^{[6]}$

\section{Objectives}

- To estimate prevalence of HER2/neu positivity in colorectal cancers.

- To assess the correlation between expression of HER2/neu with histological grades and stages of colorectal carcinoma.

\section{MATERIALS AND METHODS \\ Study Design}

A descriptive study of all cases of adenocarcinoma of colon or rectum during a period of 18 months from January 2014 to June 2015 was conducted in the Department of Pathology, Govt. T D Medical College, Alappuzha. 


\section{Subjects}

\section{Inclusion Criteria}

All surgically resected specimens of colorectal carcinoma with a histological diagnosis of adenocarcinoma of colon/rectum received in the Department of Pathology during the study period.

\section{Exclusion Criteria}

1. Those who received chemotherapy or radiotherapy, prior surgery.

2. Colonoscopic biopsy specimens.

\section{Sample Size}

50 cases of histopathologically proven colon/rectal carcinomas satisfying inclusion and exclusion criteria selected as conveniently.

\section{Methods}

Patient data including name, age, IP number, history of adjuvant therapy, CT scan report and other investigations, if any, were collected from the Department of Surgery and the surgery requisition form.

\section{Histopathology}

The tissue samples from primary tumour were fixed in $10 \%$ formalin for 24 hours before processing it to be embedded on paraffin blocks. Routine sections were taken and stained with Haematoxylin and Eosin. The cases with diagnosis of adenocarcinoma of colon/rectum were recruited into the study. These cases were further graded using the following grading system.

\section{Well Differentiated}

More than 95\% glands formation.

\section{Moderately Differentiated}

$50-95 \%$ glands formation.

\section{Poorly Differentiated}

Less than $50 \%$ glands with predominant solid areas.

\section{Immunohistochemistry}

Representative $3 \mu \mathrm{m}$ sections were taken from each paraffin block and immunohistochemical staining with HER2/neu was performed.

Invasive breast carcinoma sections were used as positive controls. Negative controls were created by omission of primary antibody and replacement with phosphate buffered saline.

\footnotetext{
The 4 Tiered Scoring System for Breast Carcinoma was Utilised-

Score 0 -undetectable or membrane staining $<10 \%$.

Score $1+$-faint membrane staining $>10 \%$.

Score 2+- weak-to-moderate complete membrane staining $>10 \%$.

Score 3+-moderate-to-strong complete membrane staining in $>30 \%$.

Score $0 / 1+$-negative HER2/neu expression.

Score 2+/3+ - positive HER2/neu expression.

Staging of tumour done with Modified Dukes staging system.
}

Stage A- limited to the mucosa.

Stage B - tumours extend through the wall.

Stage C1-only regional lymph nodes were positive.

Stage C2- nodes at the point of mesenteric blood vessel ligature were involved.

Stage D- presence of distant metastasis.

\section{Statistical Analysis}

Expression of HER2/neu will be assessed with respect to staging by using appropriate statistical test such as Chi square test. All statistical analysis will be carried out using SPSS software version 16.

\section{Ethical Considerations}

Study will be conducted on specimen coming routinely to Pathology Department. Consent is being routinely taken. The extra tissue section studies involve no expense to the patient. No other ethical issues involved.

\section{RESULTS}

A total of 50 colorectal carcinoma cases were included in the study. The median age of our patients, of whom 25 were male, was 60.04 years (Range 35 - 85). A peak incidence was seen in the $5^{\text {th }}$ to $6^{\text {th }}$ decades of life.

The lesions were present in the colon in 30 cases and in the rectum in 20 cases. All the cases were conventional adenocarcinomas, out of which 40 cases were of welldifferentiated (Grade 1) tumours, 8 cases were of moderately differentiated (Grade 2) tumours and 2 cases were of poorly differentiated (Grade 3) tumours. [Table 1].

The staging was done by Modified Dukes staging system and majority of patients were included in the stage B $(62 \%)$. No cases were included in the Stage A. $24 \%$ of cases were included in the stage C and $14 \%$ cases were in the stage D. [Table 2]

Immunohistochemical staining for HER2/neu was performed on all the 50 cases of colorectal carcinoma, the scoring was done and the results were interpreted in terms of the intensity, pattern and the percentage of HER2/neu staining.

There were 22 (44\%) HER2/neu positive cases and 28 (56\%) HER2/neu negative cases. $12 \%$ showed $3+$ staining, $12 \%$ showed $2+$ staining and $20 \%$ of the cases showed $1+$ positivity. Majority of the cases show membranous and cytoplasmic staining.

No cases showed pure membranous staining. HER2/neu scoring was done similar to breast carcinoma. Score 0 and $1+$ were taken as negative and Scores $2+$ and $3+$ were taken as positive. Of the total 40 cases of grade I conventional adenocarcinoma, 11 cases showed positivity, whereas in grade II, out of 8 cases, none of the cases showed positivity and there were only two cases in grade III, one of which showed 2+ positivity. Statistical analysis of grade of the tumour and HER2/neu score with Chi square analysis showed $P$ value of 0.171 . [Table 3].

Eighteen percent of stage B cases showed HER2/neu positivity. Only $4 \%$ and $2 \%$ cases respectively from stage C and stage D cases showed positivity. The stage of the tumours and HER2/neu scores were not statistically significant with a $P$ value of 0.567 . [Table 4]. 
Among 50 cases, $32 \%$ of cases showed lymph node metastasis, only $4 \%$ of cases with lymph node metastasis showed HER2/neu positivity, while $20 \%$ of cases without metastasis to lymph node gave HER2/neu positivity. This showed no significant correlation with a p value of 0.192 . [Table 5].

\begin{tabular}{|c|c|c|}
\hline Histological Grade & No. of Cases & Percentage \\
\hline Well-differentiated & 40 & $80 \%$ \\
\hline Moderately differentiated & 8 & $16 \%$ \\
\hline Poorly differentiated & 2 & $4 \%$ \\
\hline Total & $\mathbf{5 0}$ & $\mathbf{1 0 0} \%$ \\
\hline \multicolumn{2}{|c|}{ Table 1. No. of Cases According to Grades }
\end{tabular}

\begin{tabular}{|c|c|c|}
\hline Stage & No. of Patients & Percentage \\
\hline A & 0 & $0 \%$ \\
\hline B & 31 & $62 \%$ \\
\hline C & 13 & $26 \%$ \\
\hline D & 6 & $12 \%$ \\
\hline \multicolumn{2}{|c|}{ Table 2. No. of Cases According to Stages } \\
\hline
\end{tabular}

\begin{tabular}{|c|c|c|c|}
\hline Grade & $\begin{array}{c}\text { HER2/neu } \\
\text { Negative }\end{array}$ & $\begin{array}{c}\text { HER2/neu } \\
\text { Positive }\end{array}$ & \\
\hline Grade 1 & $29(58 \%)$ & $11(22 \%)$ & \\
\hline Grade 2 & $8(16 \%)$ & 0 & $\mathrm{P} 0.171$ \\
\hline Grade 3 & $1(2 \%)$ & $1(2 \%)$ & \\
\hline Total & $\mathbf{3 8 ( 7 6 \% )}$ & $\mathbf{1 2}(\mathbf{2 4} \%)$ & \\
\hline \multicolumn{4}{|c|}{$\begin{array}{r}\text { Table 3. Comparison of Grade } \\
\text { of the Tumours with HER2/neu }\end{array}$} \\
\hline
\end{tabular}

\begin{tabular}{|c|c|c|c|}
\hline Stage & HER2 Negative & HER2 Positive & \\
\hline A & 0 & 0 & \\
\hline B & $22(44 \%)$ & $9(18 \%)$ & P 0.567 \\
\hline C & $11(22 \%)$ & $2(4 \%)$ & \\
\hline D & $5(10 \%)$ & $1(2 \%)$ & \\
\hline \multicolumn{4}{|c|}{$\begin{array}{c}\text { Table 4. Comparison of Stage } \\
\text { of the Tumours with HER2/neu Score }\end{array}$} \\
\hline
\end{tabular}

\begin{tabular}{|c|c|c|c|}
\hline & HER2 Negative & HER2 Positive & \\
\hline No metastasis & $24(48 \%)$ & $10(20 \%)$ & P 0.192 \\
\hline Metastasis & $14(28 \%)$ & $2(4 \%)$ & \\
\hline Total & $\mathbf{3 8}$ & $\mathbf{1 2}$ & \\
\hline \multicolumn{4}{|c|}{$\begin{array}{c}\text { Table 5. Comparison of HER2/neu } \\
\text { with Lymph Node Metastasis }\end{array}$} \\
\hline
\end{tabular}

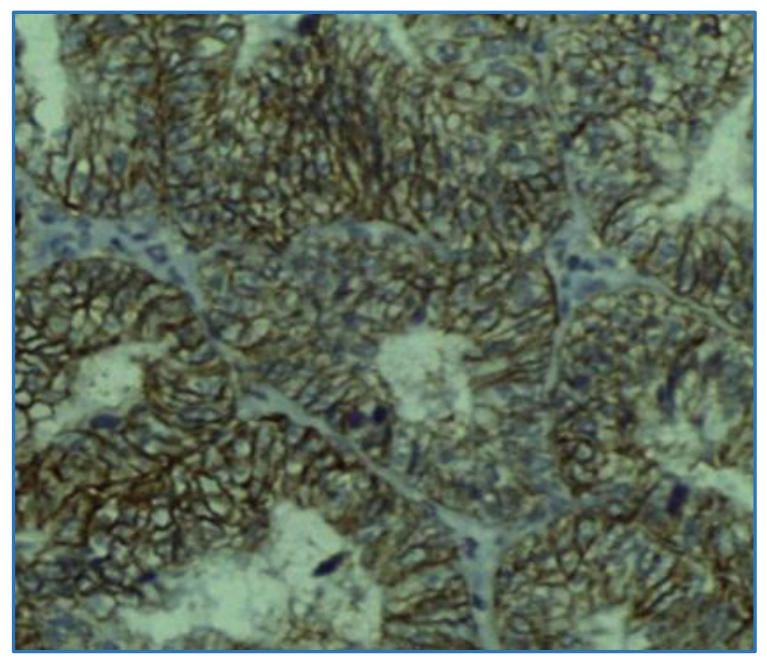

Figure 1. Colon Adenocarcinoma, HER2/neu Staining Membranous 10x

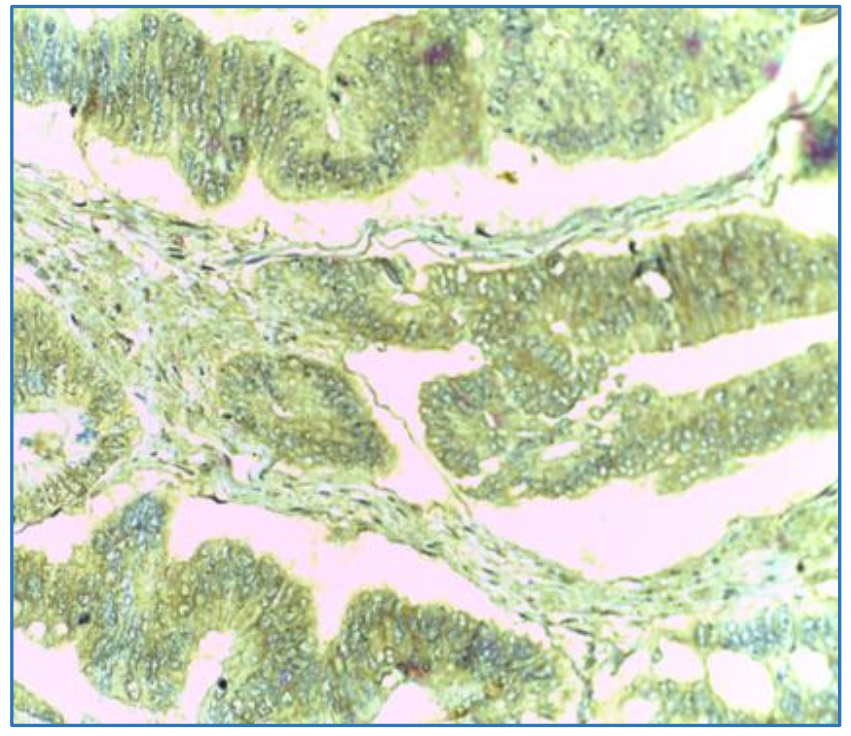

Figure 2. Colon Adenocarcinoma, HER2/neu Staining Cytoplasmic, 10x

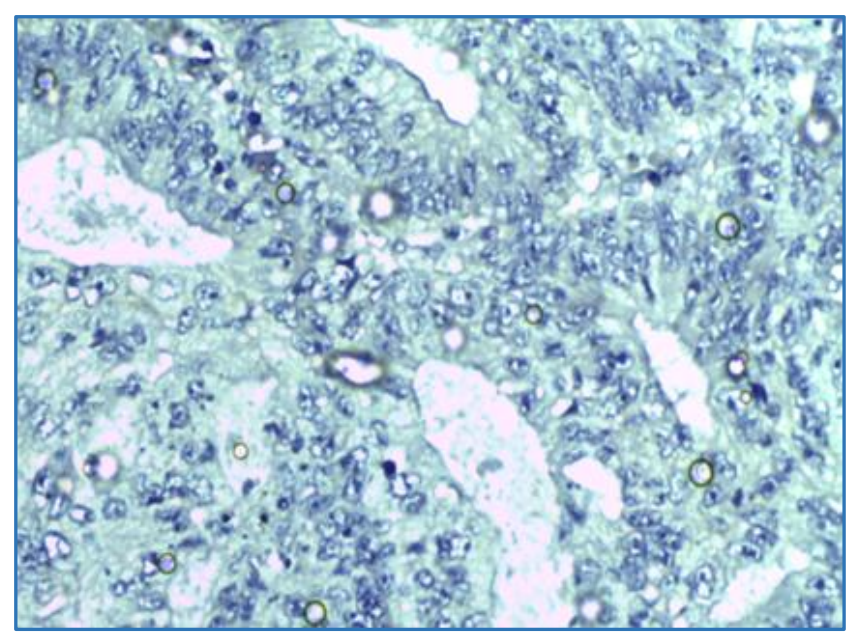

Figure 3. Colon Adenocarcinoma, HER2/neu Staining Negative, 10x

\section{DISCUSSION}

Colon cancer is one of the leading cause of cancer deaths worldwide and it constitutes $15 \%$ of all cancers. [7] HER2/neu overexpression is associated with poor prognosis and is used as a marker to predict the outcome of colon cancers.

HER2/neu can also be used to predict the patients' response to adjuvant therapy. The patients who overexpress HER2/neu should respond to Trastuzumab therapy.[8,9]

Overexpression of the HER2/neu receptor is detected in $25-35 \%$ of human breast cancers. ${ }^{[3,4]}$ But the level and incidence of HER2 overexpression in primary colon tumours appears to be different than those observed in breast cancer. Conflicting data exist about the prevalence of HER2/neu overexpression in colorectal cancer which ranges from 0 to $83 \% .{ }^{[10]}$

In this study, thirty eight cases (76\%) were HER2/neu negative and twelve cases $(24 \%)$ were HER2 positive. One of the important features of HER2/neu staining in colorectal carcinoma in our study was the pattern of staining. There was $44 \%$ of positive staining cases (including $1+$ cases), out of which 14 cases showed cytoplasmic staining and 8 cases showed cytoplasmic and 
membranous pattern of staining.

No cases showed a pure membranous pattern of staining. The intensity of staining in most of the cases varies from weak to moderate. Although cytoplasmic staining of the tumour cells was observed, but only membranous staining was considered to be specific.

Similar study done by Ghaffarzadegan et al[11] on 69 cases of colon adenocarcinoma showed 41 (59.4\%) HER2 positive and $28(40.6 \%)$ HER2 negative cases.

Twenty seven (65.9\%) cases had cytoplasmic and 14 (34.1\%) cases had membranous (predominant) and cytoplasmic staining. There was no case with pure membranous staining.

Half et al[12] discovered membranous overexpression in only five out of 96 colorectal tumours (5\%), while they determined cytoplasmic overexpression in 61 tumours (63\%).

Four out of five membranous HER2-overexpressing tumours showed amplification with FISH, while no cytoplasmic HER2-overexpressing tumours were confirmed with this technique.

Moreover, RT-PCR was performed and showed a 12fold higher mRNA expression in membranous overexpressing tumours compared to cytoplasmic overexpressing tumours.

The present study showed that well-differentiated carcinoma was more encountered than others representing $80 \%$ of cases. This was compared to the study by Ghaffarzadegan et al[11] that $75 \%$ were well differentiated. While Dalal A Elwy et al[13] found that commonest grade was moderately differentiated representing $75 \%$. In this study, most of HER2/neu positivity was noted in welldifferentiated tumours. Out of 2 poorly differentiated carcinoma cases one showed $2+$ positivity. All the cases of moderately differentiated tumours were HER2/neu negative.

Thirty one cases (62\%) of this study were stage B, 26\% cases were stage $\mathrm{C}$ and $12 \%$ cases were Stage D. This was comparable to study by Ghaffarzadegan et al[11] and they found that stage B was the most common (47\%).

Stage of the tumour was correlated with HER2/neu expression with Chi square test and it showed no significance with a $\mathrm{p}$ value of $0.567(\mathrm{P}>0.005)$. This was comparable to study by Pappas et al[14] and Kifah Hamdan Abdul Ghafour et al[15] who found that no correlation exists between these two variables.

In this study, 16 cases (32\%) showed lymph node metastasis and only 2 (4\%) cases showed HER2/neu positivity while $20 \%$ cases without lymph node metastasis showed HER2/neu positivity.

HER2/neu expression was compared with lymph node involvement and it showed no statistically significant relation with $\mathrm{p}$ value 0.192 .

This agreed with results obtained by McKay et al ${ }^{[16]}$ who found that HER2/neu was expressed in $81.8 \%$ of tumours. But they did not find any correlation between HER2/neu staining.

According to the literature, HER2/neu expression in colorectal carcinomas were variable. The broad range of the HER2/neu overexpression might be due to
1. The varying patterns of staining (cytoplasmic/membranous/both) by which overexpression was defined.

2. Difference in the fixation of the tumour tissues.

3. The diversity of the antibody and the IHC procedure which was used and lack of standardisation of the detection system. Gene amplification technique like FISH or PCR may be needed to know the exact expression of HER2/neu in colorectal carcinomas.

\section{CONCLUSION}

Overexpression of the HER2/neu receptor is detected in 25 $35 \%$ of human breast cancers ${ }^{[4,7]}$ but conflicting data exist about the prevalence of HER2/neu overexpression in colorectal cancer ranging from 0 to $83 \%$.

In our study, we analysed 50 specimens of malignant colorectal cancer lesions. In only $24 \%$ (12 specimens), the HER2/neu protein expression was observed in the therapeutic range $(2+$ and $3+), 76 \%$ were HER2/neu negative.

There was no significant association with tumour grade, stage and other clinical parameters. These data indicate that HER2/neu is unlikely to play a major role in the therapeutic management of colorectal cancer.

\section{REFERENCES}

[1] Siegel R, Desantis C, Jemal A, et al. Colorectal cancer statistics, 2014. CA cancer Clin 2014;64(2):104-17.

[2] Blok EJ, Kuppen PJK, Van Leeuwen JEM, et al. Cytoplasmic overexpression of HER2: a key factor in colorectal cancer. Clinical Medicine Insights: Oncology 2013;7:41-51.

[3] Slamon DJ, Godolphin W, Jones LA, et al. Studies of the HER2/neu proto-oncogene in human breast and ovarian cancer. Science 1989;244(4905):707-12.

[4] Slamon DJ, Clark GM, Wong SG, et al. Human breast cancer: correlation of relapse and survival with amplification of the HER2/neu oncogene. Science 1987;235(4785):177-82.

[5] Bang YJ, Cutsem VE, Feyereislova A, et al. Trastuzumab in combination with chemotherapy versus chemotherapy alone for treatment of HER2-positive advanced gastric or gastro-oesophageal junction cancer (ToGA): a phase 3, open-label, randomised controlled trial. Lancet 2010;376(9742):687-97.

[6] Ross JS, McKenna BJ. The HER2/neu oncogene in tumours of the gastrointestinal tract. Cancer Invest 2001;19(5):554-68.

[7] Gill MK, Jain $\mathrm{K}$, Manjari M, et al. Expression of HER2/neu in colon carcinoma and its correlation with the histological grades and the lymph nodes status. Journal of Clinical and Diagnostic Research 2011;5(8):1564-8.

[8] Slamon DJ, Leyland-Jones B, Shak S, et al. Use of chemotherapy plus a monoclonal antibody against HER2 for metastatic breast cancer that overexpresses HER2. N Engl J Med 2001;344:783-92. 
[9] Vogel CL, Cobleigh MA, Tripathy D, et al. Efficacy and safety of trastuzumab as a single agent in the first line treatment of HER2-overexpressing metastatic breast cancer. J Clin Oncol 2002;20(3):719-26.

[10] Neklason DW, Kerber RA, Nilson DB, et al. Common familial colorectal cancer linked to chromosome 7q31: a genome-wide analysis. Cancer Research 2008;68(21):8993-7.

[11] Ghaffarzadegan K, Sharifi N, Vosooghynia H, et al. HER2/neu expression in colon adenocarcinoma and its correlation with clinicopathologic variables. IJBMS 2006;1:64-9.

[12] Half E, Broaddus R, Danenberg KD, et al. HER2 receptor expression, localization, and activation in colorectal cancer cell lines and human tumours. Int J Cancer 2004;108(4):540-8.
[13] Elwy DA, El-Aziz A, El-Sheikh S, et al. Immunohistochemical expression of HER2/neu in colorectal carcinoma. Med J Cairo Univ 2012;80(2):467-77.

[14] Pappas A, Lagoudianakis E, Seretis C, et al. Clinical role of HER2/neu in colorectal cancer. J BUON 2013;18(1):98-104.

[15] Hamdan K, Ghafour A. Immunohistochemical expression of Her2/Neu receptor in human colorectal carcinoma. The Iraqi postgraduate medical journal 2014;13(3):424-9.

[16] McKay JA, Loane JF, Ross VG, et al. C-erbB2 is not a major factor in the development of colorectal cancer. Br J Cancer 2002;86(4):568-73. 